\title{
Formal Total Synthesis of the Polyketide Macrolactone
}

\section{Narbonolide}

Lakshmanan Venkatraman, Courtney C. Aldrich, David H. Sherman, and Robert A. Fecik*

\section{Table of Contents.}

General Procedures S2

Experimental procedures and spectral data:

Aldehyde 15 S3

PMB ether 16 S3

Alcohol 17 S4

Aldehyde 18 S4

Enone 21 S5

Carboxylic acid $\mathbf{2 8}$ S5

Alcohol 30 S6

Aldehyde 31 S7

Alcohol 36 S7

${ }^{1} \mathrm{H}$ and ${ }^{13} \mathrm{C}$ NMR spectra:

Aldehyde 15 S9

PMB ether 16 S10

Alcohol 17 S11

Aldehyde 18 S12

Alcohol 20 S13 
Enone 21

Alcohol 26

S15

Acetonide 27

S16

Carboxylic acid $\mathbf{2 8}$

S17

Ester 29 S18

Alcohol 30 S19

Aldehyde 31 S20

Allylic alcohol 32 S21

Enone 33 S22

(3S)-3-Dihydronarbonolide (8) S23

Vinyl iodide 35 S24

Alcohol 36 S25

References S26

General Procedures. All commercial reagents were used as provided unless otherwise indicated. The Dess-Martin periodinane ${ }^{1}$ was synthesized according to the modified method of Ireland, ${ }^{2}$ and $\mathrm{Zn}\left(\mathrm{BH}_{4}\right)_{2}$ was prepared according to the method of Gensler. ${ }^{3} \mathrm{THF}$ and $\mathrm{Et}_{2} \mathrm{O}$ were distilled from $\mathrm{Na} / \mathrm{benzophenone}$, and $\mathrm{CH}_{2} \mathrm{Cl}_{2}$ was distilled from $\mathrm{CaH}_{2}$. All reactions were performed under an inert atmosphere of dry $\mathrm{N}_{2}$ in oven-dried $\left(150^{\circ} \mathrm{C}\right)$ glassware. Optical rotations were determined on a polarimeter using the sodium $\mathrm{D}$ line $(\lambda=589 \mathrm{~nm})$ at the temperature indicated and are reported as follows: $[\alpha]_{\mathrm{D}}^{\text {temp }}$, concentration $(c=$ $\mathrm{g} / 100 \mathrm{~mL}$ ), and solvent. ${ }^{1} \mathrm{H}$ and ${ }^{13} \mathrm{C} \mathrm{NMR}$ spectra were recorded on a 300, 500, or $600 \mathrm{MHz}$ spectrometer. Proton chemical shifts are reported in ppm from an internal standard of residual chloroform (7.26 ppm), and carbon chemical shifts are reported using an internal standard of residual chloroform (77.0 ppm). Proton chemical data are reported as follows: chemical shift, multiplicity (ovlp = overlapping, $\mathrm{s}=$ singlet, $\mathrm{d}=$ doublet, $\mathrm{t}=$ triplet, $\mathrm{q}=$ quartet, $\mathrm{p}=$ pentet, $\mathrm{m}=$ multiplet, $\mathrm{br}=$ broad), 
coupling constant, and integration. IR spectra were recorded on an FT-IR spectrophotometer. High resolution mass spectra were obtained on an ESI-TOF/MS using either PEG or PPG standards as high resolution calibrants.

$\left(\mathbf{2 R , 4 S ) - 5 - A c e t o x y - 2 , 4 , - d i m e t h y l p e n t a n a l ~ ( 1 5 ) . ~ T o ~ a ~ s o l u t i o n ~ o f ~ a l c o h o l ~} \mathbf{1 4}^{4}(231 \mathrm{mg}, 1.14 \mathrm{mmol})\right.$ in $\mathrm{CH}_{2} \mathrm{Cl}_{2}(10 \mathrm{~mL})$ at room temperature was added the Dess-Martin periodinane $(630 \mathrm{mg}, 1.49 \mathrm{mmol}, 1.3$ equiv.) and $\mathrm{NaHCO}_{3}(287 \mathrm{mg}, 3.42 \mathrm{mmol}, 3.0$ equiv.). The reaction was stirred for $30 \mathrm{~min}$ and quenched with $1 M$ aqueous $\mathrm{Na}_{2} \mathrm{~S}_{2} \mathrm{O}_{3}(10 \mathrm{~mL})$ and saturated aqueous $\mathrm{NaHCO}_{3}(10 \mathrm{~mL})$. The biphasic mixture was stirred vigorously for $15 \mathrm{~min}$. The layers were separated and the aqueous layer was extracted with $\mathrm{CH}_{2} \mathrm{Cl}_{2}(2 \times 15 \mathrm{~mL})$. The combined organic layers were dried $\left(\mathrm{Na}_{2} \mathrm{SO}_{4}\right)$, filtered, and concentrated onto Celite under reduced pressure. Purification by flash chromatography (10\% pentane/ $\left.\mathrm{Et}_{2} \mathrm{O}\right)$ afforded the title compound $(172 \mathrm{mg}, 75 \%$ yield) as a colorless oil whose spectral data corresponded to those reported, ${ }^{5}$ and was used as such for the next reaction.

$(2 S, 4 R)-5-(4-M e t h o x y b e n z y l o x y)-2,4-d i m e t h y l p e n t y l$ acetate (16). To a solution of $p$ methoxybenzyl alcohol (7.48 mL, $60.0 \mathrm{mmol}, 2.0$ equiv.) in $\mathrm{Et}_{2} \mathrm{O}(50 \mathrm{~mL})$ at $23{ }^{\circ} \mathrm{C}$ was added $\mathrm{NaH}$ (0.144 g, $6.0 \mathrm{mmol}, 0.2$ equiv.), and the mixture was stirred for $30 \mathrm{~min}$. The solution was cooled to $0{ }^{\circ} \mathrm{C}$, trichloroacetonitrile $(6.0 \mathrm{~mL}, 60.0 \mathrm{mmol}, 2.0$ equiv. $)$ was added, and the reaction was slowly warmed to room temperature and stirred for $4 \mathrm{~h}$. The solvent was removed under reduced pressure and the residue was dissolved in petroleum ether $(50 \mathrm{~mL})$. $\mathrm{MeOH}(225 \mu \mathrm{L})$ was added to the solution, the resulting solids were removed by filtration, and the solution was concentrated under reduced pressure to obtain the imidate ether as a yellow residue. To a solution of the imidate ether in $\mathrm{Et}_{2} \mathrm{O}(40 \mathrm{~mL})$ was added monoacetate $\mathbf{1 4}^{4}(5.05 \mathrm{~g}, 30 \mathrm{mmol})$ and CSA $(50 \mathrm{mg}, 0.22 \mathrm{mmol})$. The reaction was stirred at room temperature for $24 \mathrm{~h}$ and quenched with the addition of saturated aqueous $\mathrm{NaHCO}_{3}(10 \mathrm{~mL})$. The layers were separated, and the aqueous layer was extracted with $\mathrm{Et}_{2} \mathrm{O}(3 \times 20 \mathrm{~mL})$. The combined organic layers were washed with $\mathrm{H}_{2} \mathrm{O}(10 \mathrm{~mL})$ and saturated aqueous $\mathrm{NaCl}(10 \mathrm{~mL})$, dried $\left(\mathrm{Na}_{2} \mathrm{SO}_{4}\right)$, filtered, and concentrated under reduced pressure. Purification by flash chromatography (10\% EtOAc/hexanes) 
afforded the title compound $\left(7.96 \mathrm{~g}, 93 \%\right.$ yield) as a colorless oil. $R_{f}=0.42(10 \%$ EtOAc/hexanes); $[\alpha]_{\mathrm{D}}^{23}=+1.9\left(c=0.79, \mathrm{CH}_{2} \mathrm{Cl}_{2}\right) ;{ }^{1} \mathrm{H} \mathrm{NMR}\left(\mathrm{CDCl}_{3}, 300 \mathrm{MHz}\right) \delta 7.23(\mathrm{~d}, J=8.4 \mathrm{~Hz}, 2 \mathrm{H}), 6.85(\mathrm{~d}, J=8.7$ $\mathrm{Hz}, 2 \mathrm{H}), 4.41(\mathrm{~s}, 2 \mathrm{H}), 3.94(\mathrm{dd}, J=5.4,10.4 \mathrm{~Hz}, 1 \mathrm{H}), 3.78-3.83$ (ovlp, 4H), 3.28 (dd, $J=5.4,9.0 \mathrm{~Hz}$, $1 \mathrm{H}), 3.18(\mathrm{dd}, J=6.0,9.0 \mathrm{~Hz}, 1 \mathrm{H}), 2.03(\mathrm{~s}, 3 \mathrm{H}), 1.76-1.96(\mathrm{~m}, 2 \mathrm{H}), 1.44(\mathrm{p}, J=6.9 \mathrm{~Hz}, 1 \mathrm{H}), 0.91-1.03$ (ovlp m, 7H); ${ }^{13} \mathrm{C} \mathrm{NMR}\left(\mathrm{CDCl}_{3}, 75 \mathrm{MHz}\right) \delta 171.3,159.2,131.0,129.2,113.9,75.6,72.9,69.6,55.5$, 38.2, 31.2, 30.3, 21.3, 18.3, 18.2; IR (thin film) 1737, $1246 \mathrm{~cm}^{-1}$; HRMS calcd for $\left(\mathrm{C}_{17} \mathrm{H}_{26} \mathrm{O}_{4}+\mathrm{Na}^{+}\right)$: 317.1729, found 317.1730.

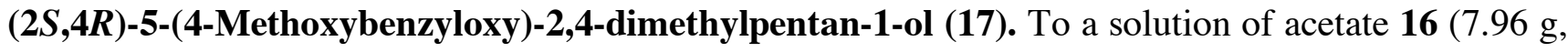
$27.1 \mathrm{mmol})$ in $\mathrm{Et}_{2} \mathrm{O}(100 \mathrm{~mL})$ at $0{ }^{\circ} \mathrm{C}$ was added $\mathrm{LiAlH}_{4}(1.54 \mathrm{~g}, 40.6 \mathrm{mmol}, 1.5$ equiv. $)$, and the mixture was slowly warmed to room temperature and stirred for $12 \mathrm{~h}$. The reaction mixture was diluted with $\mathrm{Et}_{2} \mathrm{O}(100 \mathrm{~mL})$, cooled to $0{ }^{\circ} \mathrm{C}$, and quenched by the sequential addition of $\mathrm{H}_{2} \mathrm{O}(1.5 \mathrm{~mL}), 10 \%$ aqueous $\mathrm{NaOH}(1.5 \mathrm{~mL})$, and $\mathrm{H}_{2} \mathrm{O}(3 \mathrm{~mL})$. The mixture was warmed to room temperature and stirred for $15 \mathrm{~min}, \mathrm{MgSO}_{4}$ was added, stirred for an additional $15 \mathrm{~min}$, filtered, and concentrated under reduced pressure. Purification by flash chromatography (30\% EtOAc/hexanes) afforded the title compound (5.73 g, $84 \%$ yield) as a colorless oil whose spectral data corresponded to those reported. ${ }^{6}$

(2S,4R)-5-(4-Methoxybenzyloxy)-2,4-dimethylpentanal (18). To a solution of alcohol 17 (5.73 g, $22.7 \mathrm{mmol})$ in $\mathrm{CH}_{2} \mathrm{Cl}_{2}(50 \mathrm{~mL})$ at room temperature was added the Dess-Martin periodinane (11.56 $\mathrm{g}$, $27.3 \mathrm{mmol}, 1.2$ equiv.) and pyridine (9.0 mL, $113.5 \mathrm{mmol}, 5.0$ equiv.). The reaction was stirred for $12 \mathrm{~h}$ and diluted with EtOAc $(100 \mathrm{~mL})$ and saturated aqueous $\mathrm{NaHCO}_{3}(30 \mathrm{~mL})$. The layers were separated, and the aqueous layer was extracted with EtOAc $(3 \times 20 \mathrm{~mL})$. The combined organic layers were washed with $1 M$ aqueous $\mathrm{Na}_{2} \mathrm{SO}_{3}(10 \mathrm{~mL})$ and saturated aqueous $\mathrm{NaCl}(10 \mathrm{~mL})$, dried $\left(\mathrm{Na}_{2} \mathrm{SO}_{4}\right)$, filtered, and concentrated under reduced pressure. Purification by flash chromatography (15\% EtOAc/hexanes) afforded the title compound (4.72 g, 83\% yield) as a colorless oil whose spectral data corresponded to those reported. ${ }^{6}$

$(2 S, 4 R, 6 E, 8 R, 9 R)-9-($ tert-Butyldimethylsilyloxy)-2,4,8-trimethyl-5-oxoundec-6-enyl acetate (21). 
To a solution of alcohol $20(73 \mathrm{mg}, 0.17 \mathrm{mmol})$ in $\mathrm{CH}_{2} \mathrm{Cl}_{2}(3 \mathrm{~mL})$ at room temperature was added the Dess-Martin periodinane (94 mg, 0.22 mmol, 1.3 equiv.) and $\mathrm{NaHCO}_{3}$ (43 mg, $0.51 \mathrm{mmol}, 3.0$ equiv.). The reaction was stirred for 30 min and quenched with $1 M$ aqueous $\mathrm{Na}_{2} \mathrm{~S}_{2} \mathrm{O}_{3}(5 \mathrm{~mL})$ and saturated aqueous $\mathrm{NaHCO}_{3}(5 \mathrm{~mL})$. The biphasic mixture was stirred vigorously for $15 \mathrm{~min}$. The layers were separated and the aqueous layer was extracted with $\mathrm{CH}_{2} \mathrm{Cl}_{2}(2 \times 10 \mathrm{~mL})$. The combined organic layers were dried $\left(\mathrm{Na}_{2} \mathrm{SO}_{4}\right)$, filtered, and concentrated onto Celite under reduced pressure. Purification by flash chromatography (5\% EtOAc/hexanes) afforded the title compound (58 mg, $79 \%$ yield) as a colorless oil. $R_{f}=0.67(15 \%$ EtOAc/hexanes $) ;[\alpha]_{\mathrm{D}}{ }^{23}=+34.3\left(c=2.00, \mathrm{CH}_{2} \mathrm{Cl}_{2}\right) ;{ }^{1} \mathrm{H} \mathrm{NMR}\left(\mathrm{CDCl}_{3}, 300 \mathrm{MHz}\right) \delta 6.93$ $(\mathrm{dd}, J=15.9,6.9 \mathrm{~Hz}, 1 \mathrm{H}), 6.13(\mathrm{~d}, J=15.9 \mathrm{~Hz}, 1 \mathrm{H}), 3.82-3.94(\mathrm{~m}, 2 \mathrm{H}), 3.55(\mathrm{q}, J=5.4 \mathrm{~Hz}, 1 \mathrm{H}), 2.90$ (hext, $J=6.9 \mathrm{~Hz}, 1 \mathrm{H}), 2.49$ (hext, $J=6.9 \mathrm{~Hz}, 1 \mathrm{H}), 2.05(\mathrm{~s}, 3 \mathrm{H}), 1.71-1.88(\mathrm{~m}, 2 \mathrm{H}), 1.44-1.51(\mathrm{~m}, 1 \mathrm{H})$, 1.31-1.40 (m, 1H), 1.10-1.20 (ovlp m, 1H), 1.10 (ovlp d, J=6.9 Hz, 3H), 1.03 (d, J = 6.9 Hz, 3H), 0.92 (ovlp d, $J=6.9 \mathrm{~Hz}, 3 \mathrm{H}), 0.89$ (ovlp s, 9H), 0.86 (ovlp t, $J=7.2 \mathrm{~Hz}, 3 \mathrm{H}), 0.04(\mathrm{~s}, 3 \mathrm{H}), 0.03(\mathrm{~s}, 3 \mathrm{H}) ;{ }^{13} \mathrm{C}$ $\mathrm{NMR}\left(\mathrm{CDCl}_{3}, 75 \mathrm{MHz}\right) \delta$ 203.3, 171.0, 150.3, 128.1, 76.4, 69.1, 41.6, 40.9, 36.9, 30.5, 26.8, 25.9, 21.1, 18.2, 17.9, 17.2, 14.4, 9.8, -4.2, -4.4; IR (thin film) 2960, 2932, 1742, 1693, 1671, $1626 \mathrm{~cm}^{-1}$; HRMS calcd for $\left(\mathrm{C}_{22} \mathrm{H}_{42} \mathrm{O}_{4} \mathrm{Si}+\mathrm{Na}^{+}\right): 421.2745$, found 421.2762.

\section{(R)-2-((4S,5R,6S)-6-((2S,4R)-5-(4-Methoxybenzyloxy)-4-methylpentan-2-yl)-2,2,5-trimethyl-1,3-}

dioxan-4-yl)propanoic acid (28). To a solution of oxazolidinone 27 (0.291 g, $0.5 \mathrm{mmol})$ in THF (8 $\mathrm{mL})$ and $\mathrm{H}_{2} \mathrm{O}(2 \mathrm{~mL})$ at $0{ }^{\circ} \mathrm{C}$ was added $30 \%$ aqueous $\mathrm{H}_{2} \mathrm{O}_{2}(0.57 \mathrm{~mL}, 5.0 \mathrm{mmol}, 10.0$ equiv. $)$ followed by aqueous $\mathrm{LiOH}(1 M, 1.5 \mathrm{~mL}, 1.5 \mathrm{mmol}, 3.0$ equiv.). After $1 \mathrm{~h}$ the solution was warmed to room temperature and stirred for an additional $6 \mathrm{~h}$. The solution was cooled to $0{ }^{\circ} \mathrm{C}$ and treated with an aqueous solution of $\mathrm{Na}_{2} \mathrm{SO}_{3}(1.5 \mathrm{M}, 10 \mathrm{~mL})$. After $10 \mathrm{~min}$, the mixture was diluted with EtOAc $(20 \mathrm{~mL})$ and acidified to $\mathrm{pH} 1$ with $1 M$ aqueous $\mathrm{HCl}$. The layers were separated and the aqueous layer was extracted with EtOAc $(3 \times 10 \mathrm{~mL})$. The combined organic layers were washed with saturated aqueous $\mathrm{NaCl}(10 \mathrm{~mL})$, dried $\left(\mathrm{Na}_{2} \mathrm{SO}_{4}\right)$, filtered and concentrated under reduced pressure. Purification by flash chromatography $\left(5 \% \mathrm{MeOH} / \mathrm{CH}_{2} \mathrm{Cl}_{2}\right)$ afforded the title compound $(0.14 \mathrm{~g}, 90 \%$ yield $)$ as a colorless oil. 
$R_{f}=0.42\left(5 \% \mathrm{MeOH} / \mathrm{CH}_{2} \mathrm{Cl}_{2}\right) ;[\alpha]_{\mathrm{D}}{ }^{23}=-29.5\left(c=1.35, \mathrm{CH}_{2} \mathrm{Cl}_{2}\right) ;{ }^{1} \mathrm{H} \mathrm{NMR}\left(\mathrm{CDCl}_{3}, 500 \mathrm{MHz}\right) \delta 7.28(\mathrm{~d}$, $J=8.5 \mathrm{~Hz}, 2 \mathrm{H}), 6.88(\mathrm{~d}, J=9.0 \mathrm{~Hz}, 2 \mathrm{H}), 4.45(\mathrm{AB}$ system, $J=12.0, \Delta v=35.0 \mathrm{~Hz}, 2 \mathrm{H}), 3.87(\mathrm{dd}, J=$ 2.0, 9.0 Hz, 1H), 3.32-3.40 (ovlp m, 6H), 3.16 (dd, J = 7.5, 9.0 Hz, 1H), 2.64-2.72 (m, 1H), 1.84-1.96 $(\mathrm{m}, 1 \mathrm{H}), 1.68-1.76(\mathrm{~m}, 1 \mathrm{H}), 1.56-1.66(\mathrm{~m}, 2 \mathrm{H}), 1.40(\mathrm{~s}, 6 \mathrm{H}), 1.27(\mathrm{~d}, J=9.0 \mathrm{~Hz}, 3 \mathrm{H}), 0.97(\mathrm{~d}, J=9.0$ $3 \mathrm{H}), 0.88(\mathrm{~d}, J=8.5 \mathrm{~Hz}, 3 \mathrm{H}), 0.78-0.86(\mathrm{~m}, 1 \mathrm{H}), 0.78(\mathrm{~d}, J=9.0 \mathrm{~Hz}, 3 \mathrm{H}) ;{ }^{13} \mathrm{C} \mathrm{NMR}\left(\mathrm{CDCl}_{3}, 75 \mathrm{MHz}\right)$ $\delta 180.2,159.1,131.1,129.3,113.9,99.5,78.6,75.5,75.3,72.8,55.6,42.4,38.8,32.4,32.0,31.5,30.3$, 19.9, 19.6, 15.3, 15.2, 5.5; IR (thin film) 1737, 1708, $1612 \mathrm{~cm}^{-1}$; HRMS calcd for $\left(\mathrm{C}_{24} \mathrm{H}_{38} \mathrm{O}_{6}+\mathrm{Na}^{+}\right)$: 445.2566, found 445.2588.

\section{$(R)-((3 R, 4 R, E)-6-I o d o-4-m e t h y l h e x-5-e n-3-y l)$} 2-((4S,5R,6S)-6-((2S,4R)-5-hydroxy-4-

methylpentan-2-yl)-2,2,5-trimethyl-1,3-dioxan-4-yl)propanoate (30). To a solution of the PMB ether 29 (0.290 g, $0.4475 \mathrm{mmol})$ in $\mathrm{CH}_{2} \mathrm{Cl}_{2}(8 \mathrm{~mL})$ and $\mathrm{H}_{2} \mathrm{O}(2 \mathrm{~mL})$ at $0{ }^{\circ} \mathrm{C}$ was added DDQ $(0.154 \mathrm{~g}, 0.671$ mmol, 1.5 equiv.). The reaction was stirred for $90 \mathrm{~min}$ and quenched by the addition of saturated aqueous $\mathrm{NaHCO}_{3}(5 \mathrm{~mL})$. The mixture was diluted with $\mathrm{CH}_{2} \mathrm{Cl}_{2}(20 \mathrm{~mL})$ and the layers were separated. The aqueous layer was extracted with $\mathrm{CH}_{2} \mathrm{Cl}_{2}(3 \times 5 \mathrm{~mL})$ and the combined organic layers were dried $\left(\mathrm{Na}_{2} \mathrm{SO}_{4}\right)$, filtered, and concentrated under reduced pressure. Purification by flash chromatography $(25 \%$ EtOAc/hexanes) afforded the title compound $(0.200 \mathrm{~g}, 87 \%$ yield $)$ as a colorless oil. $R_{f}=0.47(25 \%$ EtOAc/hexanes); $[\alpha]_{\mathrm{D}}^{23}=+18.1\left(c=0.80, \mathrm{CH}_{2} \mathrm{Cl}_{2}\right) ;{ }^{1} \mathrm{H} \mathrm{NMR}\left(\mathrm{CDCl}_{3}, 500 \mathrm{MHz}\right) \delta 6.45(\mathrm{dd}, J=8.5$, $14.0 \mathrm{~Hz}, 1 \mathrm{H}), 6.10(\mathrm{~d}, J=14 \mathrm{~Hz}, 1 \mathrm{H}), 4.74-4.78(\mathrm{~m}, 1 \mathrm{H}), 3.85(\mathrm{dd}, J=17.5,2.0 \mathrm{~Hz}, 1 \mathrm{H}), 3.57(\mathrm{dd}, J=$ 5.5, 10.5 Hz, 1H), 3.39-3.45 (ovlp m, 2H), 2.62-2.68 (m, 1H), 2.48 (sext, J= 7.0 Hz, 1H), 2.15 (br s, 1H), 1.53-1.72 (m, 7H), $1.43(\mathrm{~s}, 3 \mathrm{H}), 1.40(\mathrm{~s}, 3 \mathrm{H}), 1.24(\mathrm{~d}, J=7.0 \mathrm{~Hz}, 3 \mathrm{H}), 1.02(\mathrm{~d}, J=7.5 \mathrm{~Hz}, 3 \mathrm{H})$, 0.86-0.94 (ovlp m, 9H), $0.80(\mathrm{~d}, J=6.5 \mathrm{~Hz}, 3 \mathrm{H}) ;{ }^{13} \mathrm{C} \mathrm{NMR}\left(\mathrm{CDCl}_{3}, 75 \mathrm{MHz}\right) \delta 174.3,147.4,99.6$, $78.8,77.3,76.4,75.3,67.4,43.9,42.9,38.3,34.0,32.4,31.8,30.1,24.6,20.0,19.2,16.3,15.9,15.0$, 10.3, 5.3; IR (thin film) 3439 (br), 1729, $1186 \mathrm{~cm}^{-1}$; HRMS calcd for $\left(\mathrm{C}_{23} \mathrm{H}_{41} \mathrm{IO}_{5}+\mathrm{Na}^{+}\right)$: 547.1896, found 547.1888 .

$(R)-((3 R, 4 R, E)-6-I o d o-4-m e t h y l h e x-5-e n-3-y l) \quad 2-((4 S, 5 R, 6 S)-2,2,5-t r i m e t h y l-6-((2 S, 4 R)-4-m e t h y l-$ 
5-oxopentan-2-yl)-1,3-dioxan-4-yl)propanoate (31). To solution of $(\mathrm{COCl})_{2}(0.196 \mathrm{~mL}, 2.25 \mathrm{mmol}$, 1.5 equiv.) in $\mathrm{CH}_{2} \mathrm{Cl}_{2}(5 \mathrm{~mL})$ at $-78^{\circ} \mathrm{C}$ was added dropwise a solution of DMSO $(0.32 \mathrm{~mL}, 4.5 \mathrm{mmol}$, 3.0 equiv.) in $\mathrm{CH}_{2} \mathrm{Cl}_{2}(5 \mathrm{~mL})$. After $15 \mathrm{~min}$ the cloudy solution was treated with a solution of alcohol 30 (0.768 g, $1.5 \mathrm{mmol})$ in $\mathrm{CH}_{2} \mathrm{Cl}_{2}(5 \mathrm{~mL})$. The reaction was stirred for $15 \mathrm{~min}$ at $-78^{\circ} \mathrm{C}$, then treated with $i$ - $\mathrm{Pr}_{2} \mathrm{NEt}\left(1.30 \mathrm{~mL}, 7.5 \mathrm{mmol}, 5.0\right.$ equiv.). After stirring for $30 \mathrm{~min}$ at $-78{ }^{\circ} \mathrm{C}$, the reaction mixture was warmed to $0{ }^{\circ} \mathrm{C}$ and stirred for $1 \mathrm{~h}$. The reaction was quenched by the addition of saturated aqueous $\mathrm{NH}_{4} \mathrm{Cl}(10 \mathrm{~mL})$ and the mixture was slowly warmed to room temperature. The layers were separated and the aqueous layer was extracted with $\mathrm{CH}_{2} \mathrm{Cl}_{2}(3 \times 10 \mathrm{~mL})$. The combined organic layers were washed with saturated aqueous $\mathrm{NaCl}(5 \mathrm{~mL})$, dried $\left(\mathrm{Na}_{2} \mathrm{SO}_{4}\right)$, filtered, and concentrated under reduced pressure. Purification by flash chromatography (10\% EtOAc/hexanes) afforded the title compound (0.68 $\mathrm{g}, 89 \%$ yield $)$ as a colorless oil. $R_{f}=0.45(10 \%$ EtOAc/hexanes $) ;[\alpha]_{\mathrm{D}}{ }^{23}=+22.8\left(c=1.60, \mathrm{CH}_{2} \mathrm{Cl}_{2}\right) ;{ }^{1} \mathrm{H}$ NMR $\left(\mathrm{CDCl}_{3}, 300 \mathrm{MHz}\right) \delta 9.49(\mathrm{~s}, 1 \mathrm{H}), 6.39(\mathrm{dd}, J=8.4,14.4 \mathrm{~Hz}, 1 \mathrm{H}), 6.05(\mathrm{dd}, J=14.4 \mathrm{~Hz}, 1 \mathrm{H})$, 4.65-4.77 (m, 1H), $3.79(\mathrm{dd}, J=10.2,1.8 \mathrm{~Hz}, 1 \mathrm{H}), 3.36(\mathrm{dd}, J=9.6,1.8 \mathrm{~Hz}, 1 \mathrm{H}), 2.52-2.64(\mathrm{~m}, 1 \mathrm{H})$, 2.38-2.50 (m, 2H), 2.06-2.12 (m, 1H), 1.44-1.62 (m, 5H), $1.36(\mathrm{~s}, 3 \mathrm{H}), 1.32(\mathrm{~s}, 3 \mathrm{H}), 1.18(\mathrm{~d}, J=8.2$ Hz, 3H), $1.04(\mathrm{dd}, J=6.9,1.8 \mathrm{~Hz}, 3 \mathrm{H}), 0.97$ (dd, $J=7.2,1.8 \mathrm{~Hz}, 3 \mathrm{H}), 0.78-0.86$ (ovlp m, 6H), 0.74 (d, $J=7.2 \mathrm{~Hz}, 3 \mathrm{H}) ;{ }^{13} \mathrm{C} \mathrm{NMR}\left(\mathrm{CDCl}_{3}, 75 \mathrm{MHz}\right) \delta 205.8,174.4,147.4,99.4,78.3,77.3,76.4,75.2,44.9$, $43.9,42.9,35.9,32.7,31.8,30.2,24.6,19.9,15.9,15.4,15.3,15.0,10.3,5.4$; IR (thin film) 2971,2936 2878, 1728, $1185 \mathrm{~cm}^{-1}$; HRMS calcd for $\left(\mathrm{C}_{23} \mathrm{H}_{39} \mathrm{IO}_{5}+\mathrm{Na}^{+}\right): 545.1740$, found 545.1759.

(3R,4R,E)-6-Iodo-4-methylhexa-1,5-dien-3-ol (36). To a solution (1 mL, prepared from 48\% HF (0.5 $\mathrm{mL}), \mathrm{MeCN}(8.5 \mathrm{~mL})$, and $\left.\mathrm{H}_{2} \mathrm{O}(0.5 \mathrm{~mL})\right)$ was added silyl ether $35(0.13 \mathrm{~g}, 0.37 \mathrm{mmol})$ and the reaction was stirred at room temperature for $3 \mathrm{~h}$. The reaction was diluted with $\mathrm{Et}_{2} \mathrm{O}(40 \mathrm{~mL})$ and washed with saturated aqueous $\mathrm{NaHCO}_{3}(5 \mathrm{~mL})$ and saturated aqueous $\mathrm{NaCl}(5 \mathrm{~mL})$. The organic layer was dried $\left(\mathrm{MgSO}_{4}\right)$, filtered, and concentrated under reduced pressure. Purification by flash chromatography (30\% EtOAc/hexanes) afforded the title compound $(0.084 \mathrm{~g}, 95 \%$ yield $)$ as a colorless oil. $R_{f}=0.63(30 \%$ EtOAc/hexanes); $[\alpha]_{\mathrm{D}}^{23}=+15.8\left(c=0.93, \mathrm{CH}_{2} \mathrm{Cl}_{2}\right) ;{ }^{1} \mathrm{H} \mathrm{NMR}\left(\mathrm{CDCl}_{3}, 300 \mathrm{MHz}\right) \delta 6.51(\mathrm{dd}, J=8.4$, 
14.4 Hz, 1H), $6.10(\mathrm{~d}, J=14.7 \mathrm{~Hz}, 1 \mathrm{H}), 5.77-5.88(\mathrm{~m}, 1 \mathrm{H}), 5.18-5.28(\mathrm{~m}, 2 \mathrm{H}), 4.01$ (br s, 1H), 2.35$2.44(\mathrm{~m}, 1 \mathrm{H}), 1.64($ br s, $1 \mathrm{H}), 1.02(\mathrm{~d}, J=6.6 \mathrm{~Hz}, 3 \mathrm{H}) ;{ }^{13} \mathrm{C} \mathrm{NMR}\left(\mathrm{CDCl}_{3}, 75 \mathrm{MHz}\right) \delta 147.8,138.1$, 116.8, 76.5, 75.9, 46.5, 14.8; IR (thin film) 3388 (br), 3075, 2966, $1602 \mathrm{~cm}^{-1}$; HRMS calcd for $\left(\mathrm{C}_{7} \mathrm{H}_{11} \mathrm{IO}\right.$ $\left.+\mathrm{NH}_{4}^{+}\right):$256.0193, found 256.0222. 
LV-II-21-2nd time

Archive directory: /data/venkat/vnm sys/data
Sample directory: II-21-II_06Apr2005

Pulse Sequence: s2pul

Solvent: CDC13

Amblent temper
File: PROTON

Mercury-30OBB "medicina 12"

Relax. delay 5.000 se
Pulse 45.0 degrees

Acq. time 1.998
Width $3601.0 \mathrm{~Hz}$

16 repetitions $30.055957 \mathrm{MHZ}$

DATA PROCESSING
FT SIZE 16384

time $2 \mathrm{~min}, 9 \mathrm{sec}$

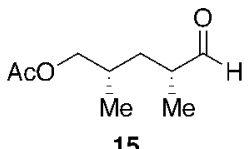

15
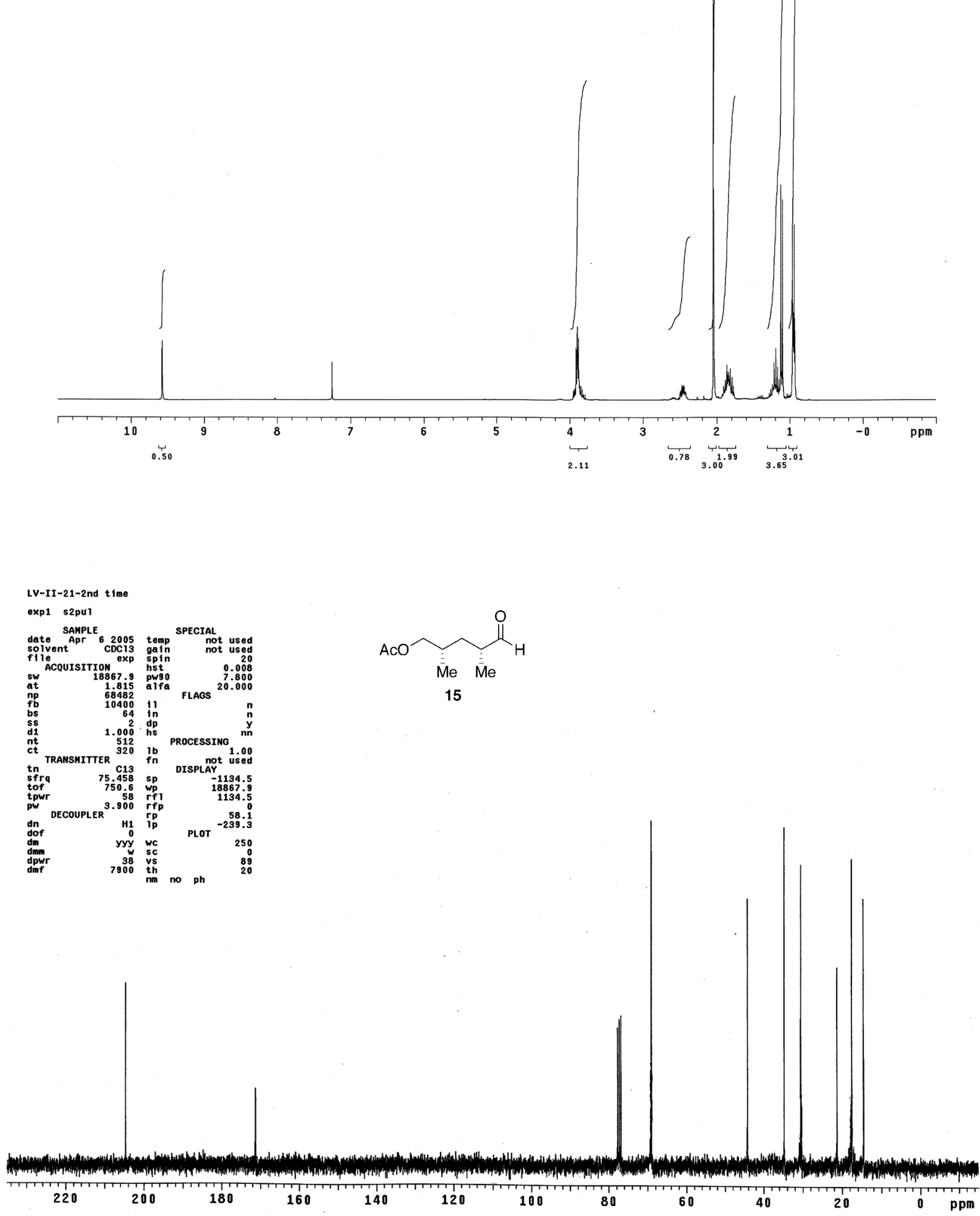
1-163-PMB-OAC

Archive directory: /data/venkat/vnmrsys/data
Sample directory: 1 1-163_14f eb2005

Pulse Sequence: s2pul

Solvent: CDC13
Ambient temperature

File: PROTON
Mercury-300BB "medicinal2"

Relax. delay $2.000 \mathrm{sec}$

Acq. time $1.998 \mathrm{sec}$

OBSERVE H1 $300.0575957 \mathrm{MHZ}$

FT size 32768

Total time $1 \mathrm{~min}, 16 \mathrm{sec}$
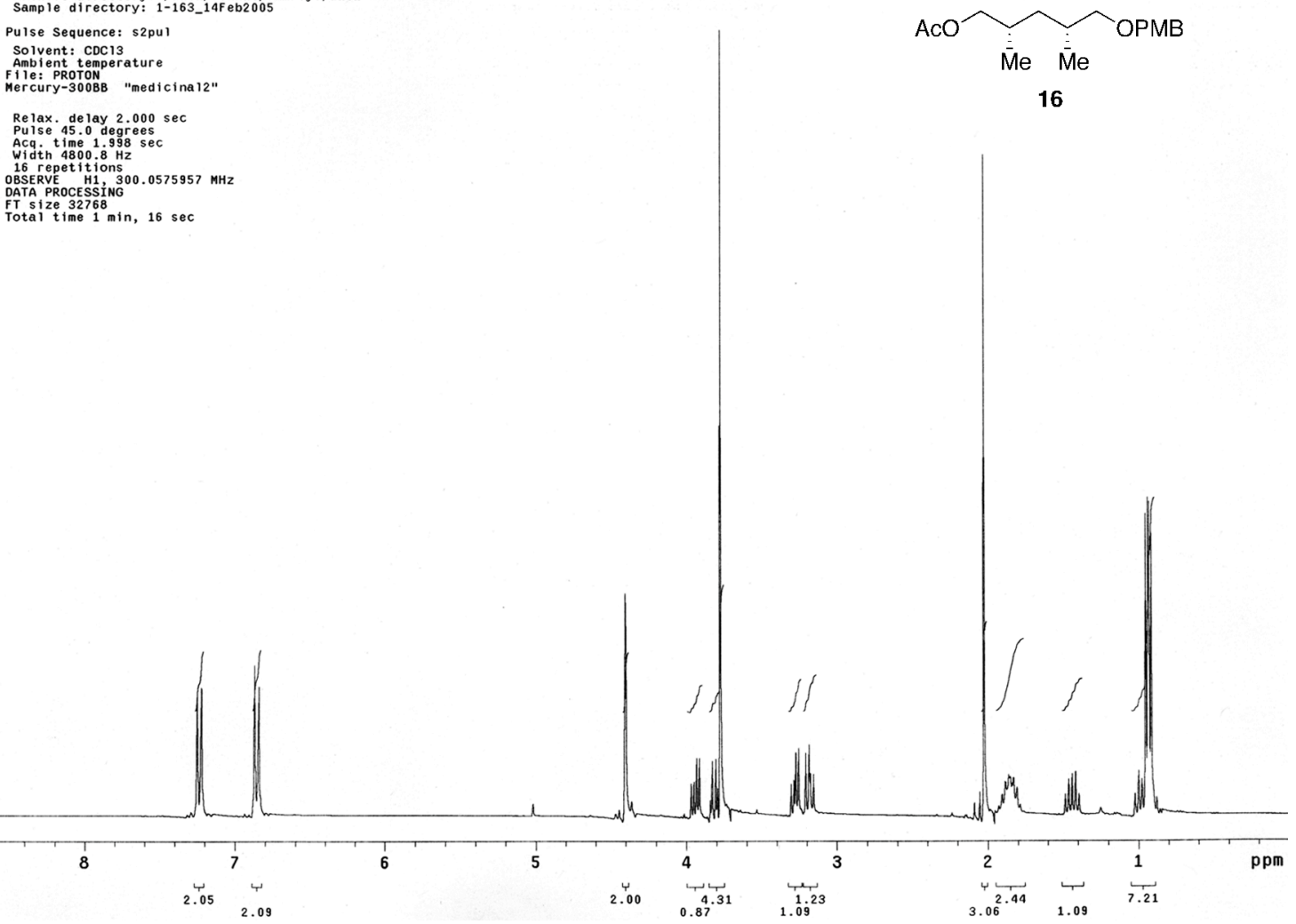

1-163-PMB-OAC

Archive directory: /data/venkat/vnmrsys/data
Sample directory: $1-163$-14f eb2005

Pulse Sequence: s2pul

Solvent: CDC13
Ambient temperature

Mercury-300B "medicinal2"

Relax. de lay $1.000 \mathrm{se}$
Pulse 45.0 degres

Acq. $t$ ime 1.815

Width $18867.9 \mathrm{~Hz}$

OBSERVE C13, 75.4495244 MHZ

Power 38 dB
cont inuous 15 on
WALTZ-16

WaLT2-16 modulated

Line broadenting $1.0 \mathrm{~Hz}$
$\mathrm{FT}$
$\mathrm{T}$

FT size $131072 \mathrm{~m} 1 \mathrm{n}, 5 \mathrm{sec}$
Total time $25 \mathrm{~min}$

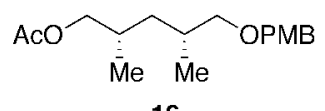

16

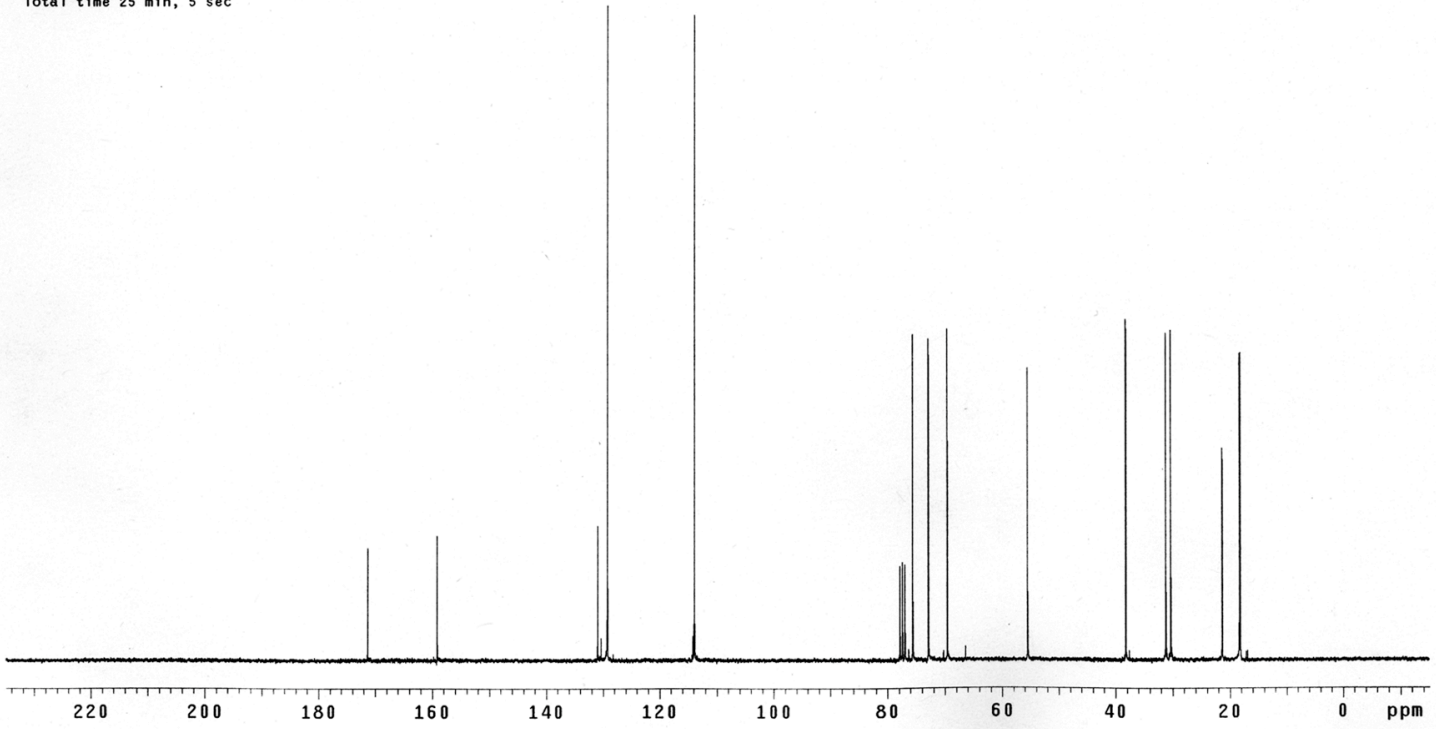




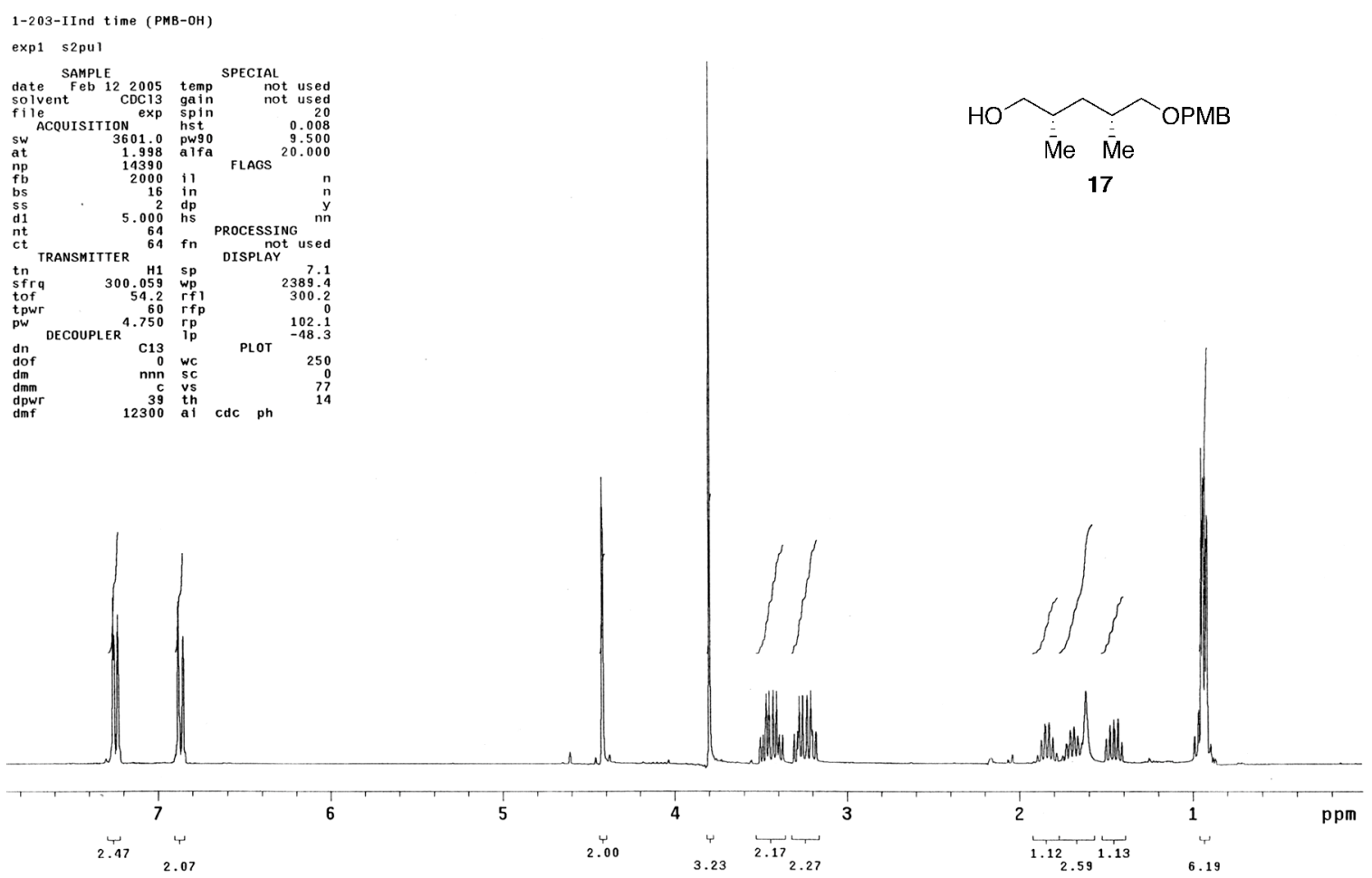

1-203-IInd time (PMB-OH)

Archive directory: /data/venkat/vnmrsys/data
Samp le directory: 1-203-II_-12F eb2005-23: $45: 37$

Pulse Sequence: s2pul

Solvent:
Ambient temperatur

Mle: CARBON
Mercury-300BB "medicina $12 "$

Relax. de lay $1.000 \mathrm{sec}$
Puise 45.0 degrees

Acq time 1.815 s

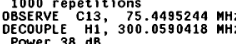

Power 38 di
cont inuous ly on

line broadenting $1.0 \mathrm{~Hz}$

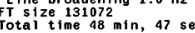

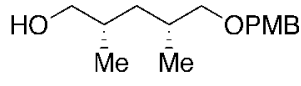

17

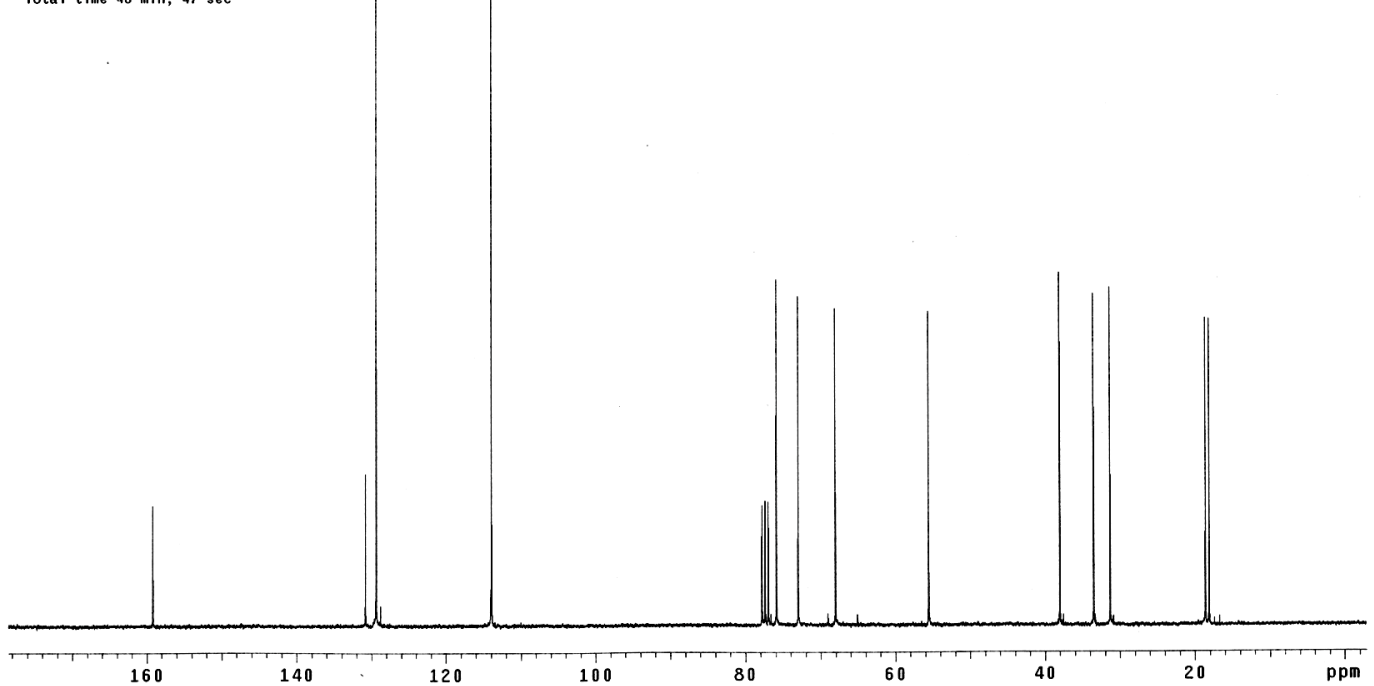


204-F 1p

Archive directory: Jdata/venkat, vnnmsys sdata
Sample directory: 204-F1_280ct2004-13:03:25

Pulse Sequence: s2pul

Solvent: CDCl $^{2}$
Amb ent temperature

Amb e PROTON
File
mercury-300B "medicinal2"

Relax. delay $5.000 \mathrm{sec}$
Pulse 45.0 degrees

Acq time $19.998 \mathrm{~s}$

Wid repetitions
16
BBSERE H1, $299.9051598 \mathrm{MHz}$

OBSERVE H1, 299.9051598
DATA PROCESSIMG

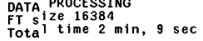
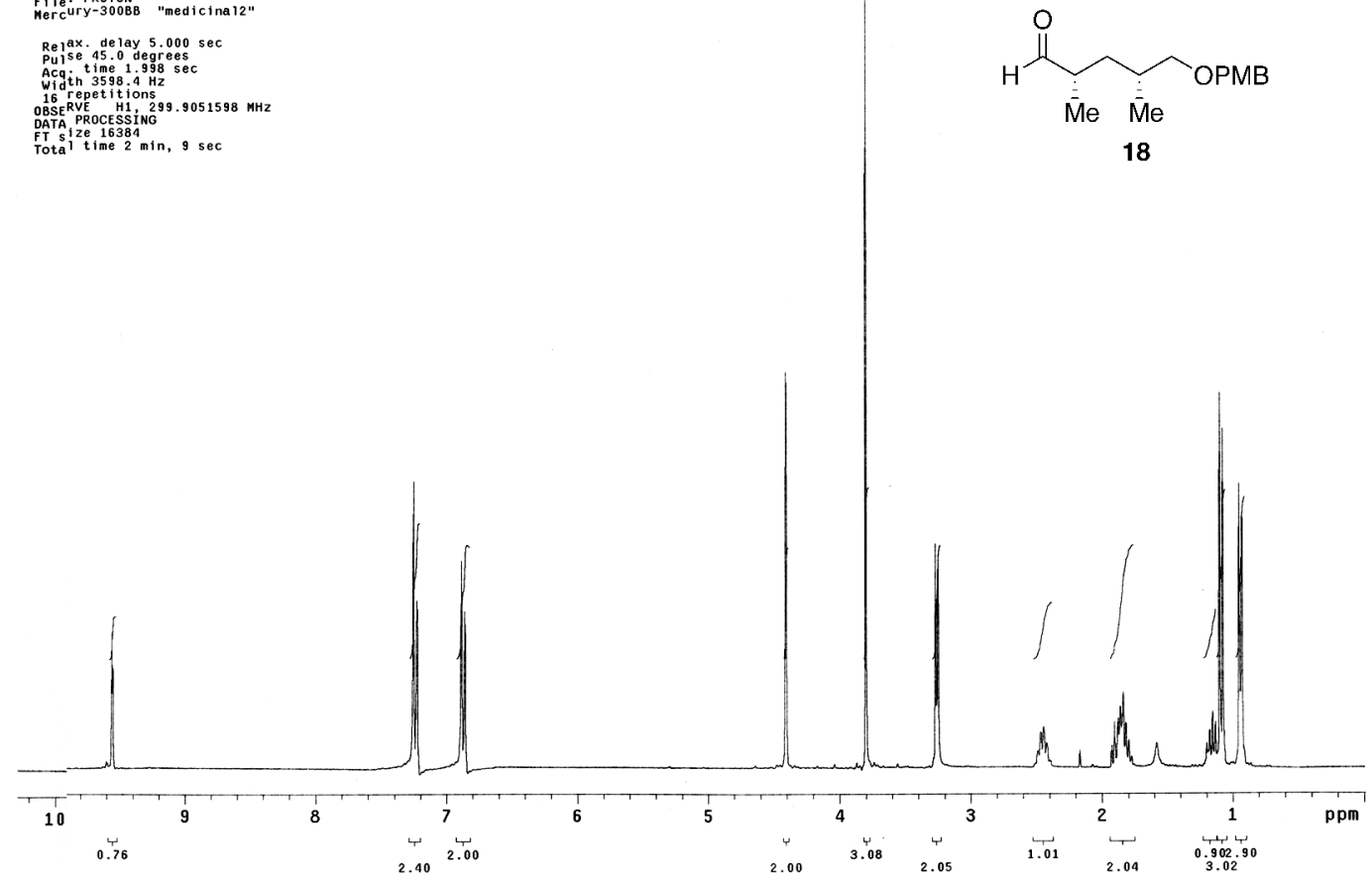

1-104-PMB (СHO)

exp1 s2pul

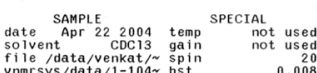

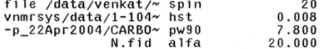

SW ACOUISIrION fid alfa FLAGS ${ }_{18867.9} 11$.

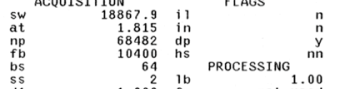

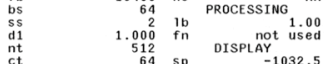

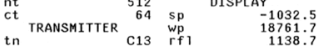

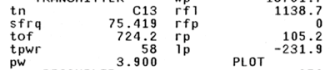

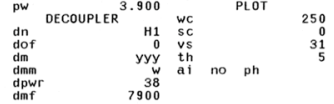
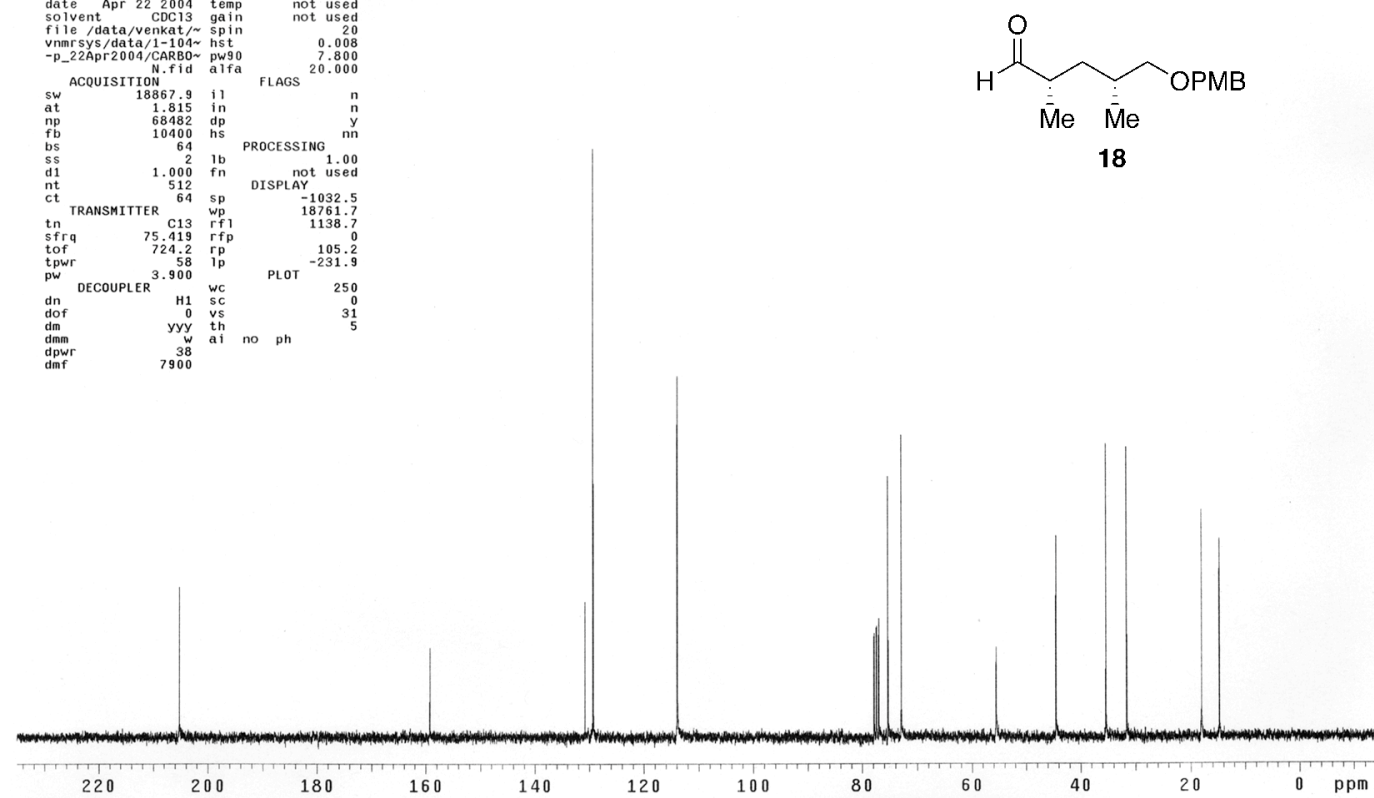